\title{
ETIOLOGY AND ANTIMICROBIAL SUSCEPTIBILITY OF NEONATAL SEPTICAEMIA IN THE NEONATAL INTENSIVE CARE UNIT IN CAN THO CHILDREN'S HOSPITAL
}

\author{
Nguyen Ngoc Rang ${ }^{1,}$, Nguyen Vi Thu Ngoc ${ }^{2}$ \\ ${ }^{1}$ Can Tho University of Medicine and Pharmacy, \\ ${ }^{2}$ Hoan My Cuu long Hospital, Can Tho
}

This study aimed to determine the causative organisms in neonatal sepsis and their antimicrobial resistance patterns in the Neonatal Intensive Care Unit (NICU) of Can Tho Children's Hospital in Vietnam. A retrospective descriptive study of neonatal sepsis was conducted from January 2018 to December 2019. A total of 139 neonates with positive blood culture was analyzed. Gram - positive bacteria $(n=84,60.4 \%)$ were more common than Gram - negative bacteria ( $n=49,35.3 \%)$ and fungi $(n=6,4.3 \%)$. Coagulase negative Staphylococci (CONS) (28.3\%) and Klebsiella pneumoniae (13.2\%) were the most common cause of Early - onset sepsis, while CONS (39.5\%) and Staphylococcus aureus (22.1\%) were predominant isolates of Late - onset sepsis. Almost CONS and S. aureus were resistant to ampicillin and oxacillin, but susceptible to vancomycin (92 - 94\%) and lizenolid (100\%). K. pneumonia was resistant to cefotaxime (67\%) and gentamicin (30\%), but susceptible to imipenem (92\%). Conclusion: CONS, S. aureus and K. pneumoniae were the most frequent pathogens in neonatal sepsis in our settings. Almost these strains were resistant to commonly used antibiotics. Change of first - line drugs should be based on the identification of isolated organisms and appropriate implementation of likely susceptible antibiotics would have a substantial impact on the outcomes.

Keywords: Neonatal sepsis, antimicrobial susceptibility, coagulase - negative Staphylococci

\section{INTRODUCTION}

Sepsis is one of the major causes of morbidity and mortality of the newborn in developing countries, where the incidence of neonatal sepsis is about 49 to 170 per 1,000 live births. ${ }^{1}$ The most common pathogens associated with neonatal sepsis in developing countries are Gram - negative organisms (Klebsiella pneumoniae, Escherichia coli, Pseudomonas spp, and Salmonella) and Gram - positive organisms (Staphylococcus aureus, coagulase - negative staphylococci (CONS),

Corresponding author: Nguyen Ngoc Rang,

Can Tho University of Medicine and Pharmacy

Email: nguyenngocrang@gmail.com

Received: 07/01/2020

Accepted: 08/03/2021
Streptococcus spp). Group B Streptococcus (GBS) is rarely encountered. ${ }^{2}$

In a previous study in Vietnam, $50 \%$ of 399 bloodstream infection isolates in neonatal sepsis were Gram - negative bacteria, most frequently K. pneumoniae (20\%), followed by Acinetobacter baumannii (15\%) and E. coli (5\%). CONS was likely to be a cause of Gram positive bacteria in $44 \%$ of isolates. None GBS was identified. ${ }^{3}$ In another cohort study which included 296 newborns infected with Gram negative bacteria, the authors observed that the organisms mainly isolated were $A$. baumannii (28\%), K. pneumonia (25\%) and Pseudomonas aeruginosa $(21 \%)$, followed by E. coli $(9 \%)$ and Serratia marcescens (3\%). Except for polymyxin $B$, these Gram - negative bacteria were highly 
resistant to many categories of antibiotics. ${ }^{4}$

The problem of inappropriate use of antibiotics has been reported in many countries, including Vietnam which has noted a rapid increase in antibiotic resistance in recent years. Many studies in Vietnam showed that the degree of resistance to antibiotics was very high in hospital settings and in the community because of poor prescribing practices and self - medication. ${ }^{5}$

This study aimed to determine the causative organisms in neonatal sepsis and their antimicrobial resistance patterns in the Neonatal Intensive Care Unit (NICU) of Can Tho Children's Hospital in Vietnam.

\section{METHODS}

\section{Study Design and Setting}

This was a retrospective descriptive study that enrolled out - born neonates admitted to NICU of Can Tho Children's Hospital between January 2018 and December 2019 with clinical signs of sepsis and positive blood culture.

Can Tho Children's Hospital with 500 - bed capacity is located in the Mekong Delta region of Vietnam. This hospital is both the teaching center of Can Tho University of Medicine and Pharmacy and the referral hospital for pediatric patients of Can Tho city and surrounding provinces of the Southwest region of Vietnam.

\section{Definitions}

Neonatal sepsis is defined as a case with clinical symptoms or perinatal risk factors and a positive culture of a single potential pathogen from the blood. Infections were classified as early - onset (EOS) if the positive culture was obtained before 72 hours of life and late - onset (LOS) if the positive blood culture was obtained after 72 hours of life. 2

\section{Study Procedures}

Blood cultures were performed in neonates with either a clinical suspicion of sepsis or risk factors for it. Sepsis was suspected in the presence of temperature instability, lethargy, poor feeding, respiratory distress, hemodynamic instability, seizure, coma, sclerema neonatorum or bleeding diathesis. Prematurity ( $<37$ weeks of gestation), low birth weight $(<2500 \mathrm{~g})$, history of resuscitation at birth, rupture of membrane (PROM) for more than 18 hours, antepartum fever, foul - smelling amniotic fluid were considered as risk factors for neonatal sepsis.

Hemocultures were done before antibiotic administration and under aseptic precautions using standard protocols for blood culture collection. All blood samples were collected for culture from peripheral line. Blood was inoculated in Soybean - Casein Digest Broth with Resins (BD Bactec Peds Plus medium). A minimum of $2 \mathrm{~mL}$ of blood was collected and inoculated as per the manufacturer's recommendations. The sample vial was transported within 30 minutes to the laboratory. Inoculated vials were placed in the BD BACTEC ${ }^{\text {TM }}$ FX40 Blood Culture System, USA. Samples entered into the fluorescent instruments were automatically analyzed based on the principle of detection of excessive production of $\mathrm{CO}_{2}$ by the instrument in vials containing viable pathogens. If no growth was obtained, the bottles were examined daily for 7 days. Positive vials were sub - cultured and a Gram - stained slide was prepared.

Bloodstream infection was diagnosed if a pathogenic organism was isolated. CoNS were regarded as contaminants if the infant was clinically good, the CRP was normal, and there were no indwelling central catheters. A repeat blood culture was required to determine whether the organism is a real pathogen or a contaminant. Organisms classified as 
contaminants were excluded from the analysis.

The antimicrobial susceptibility profile of the enterobacteria was determined using a VITEK 2 (bioMeriéux - France). For non - fermentative Gram - negative bacteria, antimicrobial susceptibility testing was performed on Mueller-Hinton agar media by standard disk diffusion method and interpreted as per Clinical and Laboratory Standard Institute guidelines. ${ }^{6}$

The empiric antibiotics of choice were cefotaxime plus ampicillin for EOS and cefotaxime (or third - generation cephalosporins) plus other antibiotics (amikacin, ciprofloxacin or vancomycin) for LOS, subsequently, the treatment was guided by the results of blood cultures.

\section{Ethical Approval}

Parents were signed informed consent forms on first attending the NICU. Ethical approval has been provided by The Science and Technology Board of Can Tho Children's Hospital and the Ethics Committee of Can Tho University of Medicine and Pharmacy.

\section{Statistical Analysis}

Data were analyzed by using Statistical Package for Social Sciences (SPSS) version 22.0 software. Categorical variables were expressed as numbers and percentages. The Pearson's Chi square test was used to compare categorical variables. Mann - Whiney tests were used for continuous variables with non normal distribution. A $P$ value of less than 0.05 was considered statistically significant.

\section{RESULTS}

\section{Characteristics of Neonates with Sepsis - Positive Blood Culture}

During the study period, there were 630 neonates admitted to the NICU with clinically suspected of sepsis. A total of 139 out - born neonates (22.0\%) had a positive blood culture, 131 (94.2\%) of them were referred from other hospitals and $8(5.8 \%)$ of them were born at home. There were 53 EOS cases and 86 LOS cases. of which 108 (81.2\%) were nosocomial infections.

The median age for EOS and LOS was 1 (1 - 2) day and 15 (8 - 20) days respectively.

In the EOS group, a lower proportion of neonates was male $(47.1 \%)$ compared to those in the LOS group $(70.9 \%)$. The proportion of low birth weight $(<2500 \mathrm{~g})$, preterm birth $(<37$ weeks), cesarean delivery, low APGAR score, premature rupture of membrane, foul - smelling of amniotic fluid, duration of hospitalization, antibiotic treatment was higher in the EOS group than that in the LOS group. The need for ventilation and inotropes, having central - line insertion and the mortality rate were higher in the EOS group than in those in the LOS group. (Table 1)

Table 1. General characteristics of the enrolled neonates

\begin{tabular}{lcccc}
\hline & $\begin{array}{c}\text { EOS } \\
\mathbf{n}=\mathbf{5 3}(\%)\end{array}$ & $\begin{array}{c}\text { LOS } \\
\mathbf{n = 8 6}(\%)\end{array}$ & $\begin{array}{c}\text { Total } \\
\mathbf{n}=\mathbf{1 3 9}(\mathbf{n} \%)\end{array}$ & p value \\
\hline $\begin{array}{l}\text { Gender } \\
\text { Male }\end{array}$ & $25(47.1)$ & $61(70.9)$ & $86(61.8)$ & 0.005 \\
Female & $28(52.8)$ & $25(29.1)$ & $53(38.2)$ & \\
\hline $\begin{array}{l}\text { Birth weight (gram) } \\
\quad 2500\end{array}$ & $26(49.0)$ & $11(13.8)$ & $37(26.6)$ & $<0.001$ \\
$\geq 2500$ & $27(51.0)$ & $75(87.2)$ & $102(73.4)$ & \\
\hline
\end{tabular}




\begin{tabular}{|c|c|c|c|c|}
\hline & $\begin{array}{c}\text { EOS } \\
\mathrm{n}=53(\%)\end{array}$ & $\begin{array}{c}\text { LOS } \\
n=86(\%)\end{array}$ & $\begin{array}{c}\text { Total } \\
n=139(n \%)\end{array}$ & $p$ value \\
\hline \multicolumn{5}{|l|}{ Preterm ( < 37 weeks) } \\
\hline Yes & $25(47.1)$ & $10(11.7)$ & $35(25.2)$ & \multirow{2}{*}{$<0.001$} \\
\hline No & $28(52.9)$ & $76(88.3)$ & $104(74.8)$ & \\
\hline \multicolumn{5}{|l|}{ Delivery } \\
\hline Vaginal & $35(66.0)$ & $71(82.5)$ & $106(76.3)$ & \multirow{2}{*}{0.026} \\
\hline Cesarean & $18(34.0)$ & $15(17.5)$ & $33(23.7)$ & \\
\hline \multicolumn{5}{|l|}{ APGAR score $<6$ at 5 minutes } \\
\hline Yes & $13(24.5)$ & $1(1.2)$ & $14(10.1)$ & \multirow{2}{*}{$<0.001$} \\
\hline No & $40(75.5)$ & $85(98.8)$ & $125(89.9)$ & \\
\hline \multicolumn{5}{|l|}{ Antepartum fever } \\
\hline Yes & $3(5.6)$ & $1(1.2)$ & $4(2.9)$ & \multirow{2}{*}{0.123} \\
\hline No & $50(94.4)$ & $85(98.8)$ & $135(97.1)$ & \\
\hline \multicolumn{5}{|l|}{ PROM of $>18$ hours } \\
\hline Yes & $12(22.6)$ & $1(1.2)$ & $13(9.4)$ & \multirow[t]{2}{*}{$<0.001$} \\
\hline No & $41(77.4)$ & $85(98.8)$ & $126(90.6)$ & \\
\hline \multicolumn{5}{|l|}{ Foul - smelling amniotic fluid } \\
\hline Yes & $8(15.1)$ & $0(0.0)$ & $8(5.8)$ & \multirow[t]{2}{*}{$<0.001$} \\
\hline No & $45(84.9)$ & $86(100)$ & $131(94.2)$ & \\
\hline \multicolumn{5}{|l|}{ Central line insertion } \\
\hline Yes & $32(60.4)$ & $11(12.8)$ & $43(30.9)$ & \multirow{2}{*}{$<0.001$} \\
\hline No & $21(39.6)$ & $75(87.2)$ & $96(69.1)$ & \\
\hline \multicolumn{5}{|l|}{ Need for ventilation } \\
\hline Yes & $30(56.6)$ & $12(13.9)$ & $42(30.2)$ & \multirow{2}{*}{$<0.001$} \\
\hline No & $23(43.4)$ & $74(86.1)$ & $97(69.8)$ & \\
\hline \multicolumn{5}{|l|}{ Need for inotropes } \\
\hline Yes & $18(33.9)$ & $9(10.5)$ & $27(19.4)$ & \multirow{2}{*}{$<.001$} \\
\hline No & $35(66.1)$ & $77(89.5)$ & $112(80.6)$ & \\
\hline $\begin{array}{l}\text { Length of hospitalization } \\
\text { (d, median, IQR) }\end{array}$ & $26(17-39)$ & $16(14-22)$ & $18(14-27)$ & $<0.001$ \\
\hline Antibiotic treatment lasted (d) & $21(15-22)$ & $16(14-22)$ & $18(14-25)$ & $<0.001$ \\
\hline \multicolumn{5}{|l|}{ Death } \\
\hline Yes & $17(32.1)$ & $12(13.9)$ & $29(20.9)$ & \multirow[t]{2}{*}{0.011} \\
\hline No & $36(67.9)$ & $74(86.1)$ & $110(79.1)$ & \\
\hline
\end{tabular}

EOS: Early-onset sepsis; LOS: Late-onset sepsis; PROM: Premature rupture of membrane

\section{Clinical Features and Hematological Parameters}

Respiratory distress $(82.7 \%)$ and lethargy $(71.9 \%)$ were the most common clinical manifestations for both the EOS and LOS groups. Jaundice (88.7\%), abdominal distention (49.1\%), tachycardia $(43.4 \%)$ occurred most commonly in the EOS group, while hypothermia $(54.0 \%)$ and poor 
feeding (43.9\%) occurred most commonly in the LOS group. Abnormal white blood cell counts, thrombocytopenia and high C - reactive protein were more observed in the EOS group. (Table 2)

Table 2. Clinical symptoms and hematological parameters in neonates with early-onset and late-onset sepsis

\begin{tabular}{|c|c|c|c|c|}
\hline & $\begin{array}{c}\text { EOS } \\
n=53(\%)\end{array}$ & $\begin{array}{c}\text { LOS } \\
n=86(\%)\end{array}$ & $\begin{array}{c}\text { Total } \\
\mathrm{n}=139(\%)\end{array}$ & $p$ value \\
\hline Fever & $10(18.9)$ & $13(15.1)$ & $23(16.5)$ & 0.563 \\
\hline Hypothermia & $19(35.8)$ & $56(65.1)$ & $75(54.0)$ & 0.001 \\
\hline Lethargy & $43(81.1)$ & $57(66.3)$ & $100(71.9)$ & 0.058 \\
\hline \multirow[t]{2}{*}{ Seizure } & $7(13.2)$ & $7(8.1)$ & $14(10.1)$ & 0.335 \\
\hline & $4(7.5)$ & $2(2.3)$ & $6(4.3)$ & 0.141 \\
\hline Respiratory distress & $51(96.2)$ & $64(74.4)$ & $115(82.7)$ & 0.001 \\
\hline Apnoea & $19(35.8)$ & $9(10.5)$ & $27(19.4)$ & $<0.001$ \\
\hline Tachycardia & $23(43.4)$ & $14(16.3)$ & 37 (26.6) & $<0.001$ \\
\hline Shock & $19(35.8)$ & $9(10.5)$ & $28(20.1)$ & $<0.001$ \\
\hline Poor feeding & $14(26.4)$ & $47(54.7)$ & $61(43.9)$ & 0.001 \\
\hline Nausea/vomiting & $3(5.7)$ & $22(25.6)$ & $25(18.0)$ & 0.003 \\
\hline Abdominal distension & $26(49.1)$ & $33(38.4)$ & $59(42.4)$ & 0.216 \\
\hline Diarrhea & $3(5.7)$ & $7(8.1)$ & $10(7.2)$ & 0.583 \\
\hline Jaundice & $47(88.7)$ & $39(45.3)$ & $86(61.9)$ & $<0.001$ \\
\hline Impetigo & $3(5.7)$ & $30(34.9)$ & $33(23.7)$ & 0.001 \\
\hline Periumbilical redness & $7(13.2)$ & $10(11.6)$ & $17(12.2)$ & 0.782 \\
\hline Slerema neonatorum & $7(13.2)$ & $4(4.7)$ & $11(7.9)$ & 0.070 \\
\hline Bleeding diathesis & $21(39.6)$ & $6(7.0)$ & $27(19.4)$ & $<0.001$ \\
\hline \multicolumn{5}{|l|}{ Hematological parameters } \\
\hline \multirow{2}{*}{ WBC $(<6$ or $>30) \times 109 / L$} & $17(32.1)$ & $5(5.8)$ & $22(15.8)$ & \multirow{2}{*}{$<0.001$} \\
\hline & $36(67.9)$ & $81(94.2)$ & $117(84.2)$ & \\
\hline \multirow{2}{*}{ Hemoglobin $(<110) \mathrm{g} / \mathrm{L}$} & $4(7.5)$ & $8(9.3)$ & $12(8.6)$ & \multirow{2}{*}{0.720} \\
\hline & $49(92.5)$ & $78(90.7)$ & $127(91.4)$ & \\
\hline \multirow{2}{*}{ Platelets $(<150) \times 109 / L$} & $22(41.5)$ & $7(8.1)$ & $29(20.9)$ & \multirow{2}{*}{$<0.001$} \\
\hline & $31(58.5)$ & $79(91.9)$ & $110(79.1)$ & \\
\hline \multirow{2}{*}{$\mathrm{CRP}(>10) \mathrm{mg} / \mathrm{L}$} & $34(64.2)$ & $25(29.1)$ & $59(42.4)$ & \multirow{2}{*}{$<0.001$} \\
\hline & $19(35.8)$ & $61(70.9)$ & $80(57.6)$ & \\
\hline
\end{tabular}

EOS: Early-onset neonatal sepsis; LOS: Late-onset neonatal sepsis; WBC: White blood cell; CRP: C-reative protein 


\section{Isolated Organisms in Neonates with EOS and LOS}

A total of $84(60.4 \%)$ cases had Gram - positive isolates, 49 (35.3\%) had Gram - negative isolates and $6(4.3 \%)$ had fungal isolates.

Table 3. Isolated organisms in 139 neonates with sepsis in NICU, Can Tho Children's Hospital, Vietnam

\begin{tabular}{lccc}
\hline & EOS & LOS & Total \\
& $\mathbf{n}=\mathbf{5 3}(\mathbf{\%})$ & $\mathbf{n = 8 6 ( \% )}$ & $\mathbf{n = 1 3 9 ( \% )}$ \\
\hline Gram positive & & & \\
CONS & & & \\
$\quad$ S. aureus & $15(28.3)$ & $34(39.5)$ & $49(35.3)$ \\
$\quad$ Streptococcus spp & $5(9.4)$ & $19(22.1)$ & $24(17.3)$ \\
$\quad$ Group B streptococcus & $1(1.8)$ & $0(0.0)$ & $1(0.7)$ \\
$\quad$ Other stretococci* & $4(7.8)$ & $6(7.0)$ & $10(7.2)$ \\
\hline Gram negative & & & $12(8.6)$ \\
$\quad$ Klebsiella pneumoniae & $7(13.2)$ & $5(5.8)$ & $11(7.9)$ \\
$\quad$ Stenotrophomonas spp & $5(9.4)$ & $6(7.0)$ & $7(5.0)$ \\
$\quad$ Acinetobacter spp & $3(5.7)$ & $4(4.7)$ & $6(4.3)$ \\
$\quad$ Escherichia coli & $4(7.5)$ & $2(2.3)$ & $2(1.4)$ \\
$\quad$ Pseudomonas spp & $1(1.9)$ & $4(4.7)$ & $6(4.3)$ \\
$\quad$ Enterobacter spp & $0(0.0)$ & $2(2.3)$ & $3(2.1)$ \\
$\quad$ Others & $3(4.3)$ & $3(3.5)$ & $3(2.1)$ \\
\hline Fungi & & & $1(1.2)$ \\
Candida albicans & $2(3.7)$ & $0(0.0)$ & \\
Kodamaea ohmeri & $3(5.7)$ & & \\
\hline
\end{tabular}

EOS: Early-onset sepsis; LOS: Late-onset sepsis; * Other stretococci: S. pneumonia (2); S. bovis (2); S. faecalis (2); S. pyogenes (1); S. milleri (1); not identified (2)

In the EOS group, the most common Gram - positive organisms isolated were CONS (28.3\%), followed by S. aureus (9.4\%) and Streptococcus spp. (9.4\%). Among Gram - negative isolates, K. pneumoniae (13.2\%) was the commonest organism isolated followed by Stenotrophomonas maltophilia (9.4\%), E. coli (7.5\%), Acinetobacter baumannii (5.7\%) and Pseudomonas spp (1\%). Fungi were isolated in 5 neonates including Kodamaea ohmeri (5.7\%) and Candida albicans (3.7\%). (Table 3).

In the LOS group, CONS (35.3\%) was the commonest Gram - positive organism followed by $S$. aureus (17.3\%) and Streptococcus spp (7.0\%). Among Gram - negative isolates, K, pneumoniae (8.6\%) was predominant organism isolated followed by S. maltophilia (7.9\%), E. coli (4.5\%), A. baumannii (5.0\%), Pseudomonas spp (3.6\%) and Enterobacter spp (1.4\%). Candida albicans was isolated in only one neonate (Table 3).

In the group with hospital - acquired infections, Gram - negative organism accounted for $66.7 \%$ $(72 / 108)$ compared with $52 \%(13 / 25)$ in the group with community - acquired infections, but the difference was not statistically significant $(p=0.081)$. 


\section{Antimicrobial Resistance of the Isolated Pathogens}

Antibacterial susceptibility tests revealed that Gram - positive isolates had the highest resistance to amoxicillin (91 - 100\%), oxacillin (89 - 100\%), but remained susceptible to vancomycin (92 - 100\%) and linezolid (100\%).

Among the Gram - negative isolates, the majority of screened isolates were resistant to amoxicillin (67 - 100\%), cefotaxime (40 - 80\%), ceftazidime (40 - 100\%) and gentamycin (30-83\%). A moderate degree of resistance to ciprofloxacin (0 - 50\%) and netilmicin (20 - 40\%) was observed among Gram - negative bacteria. Imipenem (92 - 100\%) showed the highest effectiveness against $K$. pneumonia and $P$. aeruginosa isolates, whereas polymyxin B (92 - 100\%) had the highest effectiveness against Enterobacter spp and $K$, pneumoniae isolates. S. maltophilia was almost resistant to all antimicrobial agents, but remained relatively susceptible to ciprofloxacin (82\%). With fungal isolates, Candida albicans showed $100 \%$ resistance to itraconazole, while $100 \%$ sensitivity to ketoconazole. Also, Kodamaea ohmeri showed $100 \%$ resistance to itraconazole but $100 \%$ sensitivity to fluconazole. (Table 4)

Table 4. Percentage of antibiotic resistance of the isolated pathogens in NICU, Can Tho Children's Hospital, Vietnam

\begin{tabular}{|c|c|c|c|c|c|c|c|c|c|c|c|c|c|c|}
\hline & AMX & AMC & CTX & CAZ & GEN & NET & AMK & CIP & IPM & PMB & OXA & CLI & VAN & LZD \\
\hline $\begin{array}{l}\text { Klebsiella } \\
\text { pneumonia }\end{array}$ & 100 & 64 & 67 & 100 & 30 & 20 & 25 & 33 & 8 & 9 & NT & NT & NT & \\
\hline $\begin{array}{l}\text { Escherichia } \\
\text { Coli }\end{array}$ & 100 & 80 & 67 & 50 & 50 & 33 & 0 & 50 & 33 & 17 & NT & NT & NT & \\
\hline $\begin{array}{l}\text { Acinetobacter } \\
\text { baumannii }\end{array}$ & 83 & 33 & 57 & 83 & 83 & 33 & 100 & 20 & 29 & 29 & NT & NT & NT & \\
\hline $\begin{array}{l}\text { Pseudomonas } \\
\text { aeruginosa }\end{array}$ & 80 & 40 & 40 & 40 & 40 & 0 & 0 & 20 & 0 & 40 & NT & NT & NT & \\
\hline $\begin{array}{l}\text { Enterobacter } \\
\text { Spp }\end{array}$ & NT & 100 & 50 & 100 & 50 & 0 & 50 & 0 & 50 & 0 & NT & NT & NT & \\
\hline S.maltophilia & 67 & 86 & 80 & 80 & 83 & 40 & 100 & 18 & 45 & 56 & NT & NT & NT & \\
\hline $\begin{array}{l}\text { Staphylococcus } \\
\text { Aureus }\end{array}$ & 100 & 46 & 40 & 33 & 82 & 9 & NT & 79 & 17 & 50 & 96 & 70 & 8 & 0 \\
\hline CONS & 91 & 48 & 33 & 33 & 42 & 6 & NT & 50 & 4 & 50 & 89 & 50 & 6 & 0 \\
\hline $\begin{array}{l}\text { Streptococcus } \\
\text { spp }\end{array}$ & 100 & 22 & 38 & NT & 91 & 10 & NT & 73 & 9 & NT & 100 & 64 & 0 & 0 \\
\hline
\end{tabular}

AMX: amoxicillin; AMC: amoxicillin-clavulanate; CTX: cefotaxime;CAZ:ceftazidime; GEN: gentamycin; NET: netilmicin; AMK: amikacin; CIP: ciprofloxacin; IPM: imipenem; PMB: polymyxin B; OXA: oxacillin; CLI: clindamycin; VAN: vancomycin; LSD: linezolid; CONS: coagulase-negative Staphylococci. NT: Not tested 


\section{DISCUSSION}

To the best of our knowledge, this is the first report of the causative pathogens of neonatal sepsis in the largest NICU of the Mekong Delta region of Vietnam. In our settings, Gram - positive infections were found to be more common than Gram - negative and fungal infections in all sepsis types. This finding was consistent with a meta - analysis of Li JY et al. ${ }^{7}$ who found that $81.5 \%$ of Gram - positive bacteria is responsible for neonatal sepsis in China.

In this cohort, sepsis in outborn neonates has several distinct features because the source of infection can be either community or acquired - hospital depending on the place of birth and prior hospitalization. However, most neonates $(94.2 \%)$ were referred from other hospitals, the organisms that cause neonatal sepsis were similar to those in nosocomial infections. Coagulase - negative Staphylococci (CONS) was the most common causative Gram - positive pathogens for early - onset sepsis (EOS). Similar results were reported in previous studies in Vietnam ${ }^{3}$ and in different regions of the world. ${ }^{8} \mathrm{~K}$. pneumoniae was the most common Gram - negative for EOS representing $13.2 \%$ of all isolates. This finding is in line with other studies from other regions. However. the most common causative pathogens (CONS, K. pneumonia) for EOS in our settings are not similar to those reported from developed countries, where Group B Streptococcus (GBS) and Escherichia coli are the main causes of EOS. ${ }^{10}$

The two predominant causative pathogens for late - onset sepsis (LOS) in the present study were CONS followed by $S$. aureus, similar to those reported in many developed countries. In particular, CONS was more observed in neonates with low birth weight ${ }^{11}$, while $S$. aureus was the most common Gram - positive organisms Asian countries. ${ }^{12}$

Interestingly, the prevalence of S. maltophilia was common in our NICU. This pathogen has not been isolated in neonatal sepsis in Vietnam. Nevertheless, these strains have been isolated in adult patients with septicemia in a hospital of infectious diseases in Hanoi, Vietnam. ${ }^{13}$ Recently, many authors have reported $S$. maltophilia infections as emerging global opportunistic pathogens with high multi - drug resistances in neonatal sepsis. ${ }^{14}$

Gram - negative bacilli accounted for $31 \%$ in neonates with LOS. K. pneumonia was more common than E. coli for Gram - negative bacteria in neonatal sepsis in our settings. This is in line with reports from many countries. ${ }^{9}$

The most common fungal infections in NICU were caused by Candida spp. A recent study in China and India revealed that Candida spp. was responsible for 10.2 and $22.7 \%$ of hospital acquired infections, respectively. ${ }^{15}$ In our study, fungi were detected in 6 neonates $(4.2 \%)$. In particular, Kodamaea ohmeri was first isolated in our settings.

In the present study, the causative pathogens of neonatal sepsis were resistant to almost first line drugs. The Gram - positive bacteria (CONS, S. aureus and Streptococcus spp) exhibited a high degree of resistance to ampicillin (91 $100 \%)$ and oxacillin (89 - 100\%). Some strains of CONS and $S$. aureus were also resistant to vancomycin (6 - 8\%); however, they remained susceptible $100 \%$ to linezolid. In earlier studies in Vietnam, the authors reported that $100 \%$ of CONS isolates were susceptible to vancomycin and the resistance rate to oxacillin was lower $(50 \%)$ than that of our study. ${ }^{3}$ This finding was consistent with studies from other countries where most of CONS strains were susceptible nearly $100 \%$ to vancomycin and linezolid, while 
the resistance rate to methicillin (or oxacillin) ranged from $50-80 \%{ }^{9,15}$

In our settings, the Gram - negative pathogens were almost resistant to the first line empirical therapy (ampicillin, cefotaxime and gentamycin) recommended by the World Health Organization (WHO), similar to the findings of previous studies in Vietnam ${ }^{3,4}$ and from other regions in developing countries. ${ }^{9} \mathrm{~K}$. pneumoniae and Pseudomonas spp remained susceptible to imipenem (92 - 100\%) and amikacin (75 - 100\%). Other Gram - negative pathogens (E. coli, Acinetobacter spp and Enterobacter spp) were increasingly resistant to imipenem (17 - 50\%). Specifically, $S$. maltophilia was multi - drug resistance, while remaining susceptible to ciprofloxacin in $82 \%$ of cases.

The practice of dispensing antibiotics without prescription at pharmacies, self - treatment with commonly used antibiotics (amoxicillin or cephalosporins) in the community and the wide availability and uncontrolled use of first - line drugs in the hospitals partly explain how the resistance rate of antibiotics is increasing in our settings. ${ }^{16}$

Although Vietnam was one of the first countries to develop Action Plan to Combat Antimicrobial Resistance (AMR) in the WHO Western Pacific region, the rate of resistance to antibiotics is still high, particularly in intensive care units. The fact that GBS incidence is very rare for EOS, that CONS is more common as a cause and that the emergence of new strains of multi - drug resistant bacteria such as $S$. maltophilia raises the question of changing first - line drugs for neonatal sepsis in our settings.

Continuation to implement an antibiotic surveillance program in the community, prompt microbial identification and implementation of appropriate antibiotics for neonatal sepsis in NICU are needed to reduce attributable newborn mortality.

The strengths of this study were documentation of clinical signs and risk factors of suspected sepsis from mothers. However, there were several limitations associated with this study. First, the current study was a retrospective, single - center study with a limited sample size. Second, informamation on antibiotic use in previous hospitals has not been recorded. Finally, some blood sample for CONS culture was performed only once; therefore, it may be difficult to distinguish causal pathogens from blood culture contaminants, however, the proportion of CONS in our study was similar to that reported by Li JY et al. and previous study in Vietnam. ${ }^{7}$

\section{CONCLUSION}

Coagulase - negative Staphylococci, Staphyloccocus aureus, and Klebsiella pneumoniae were prominent organisms that exhibit the highest degree of resistance to commonly used antibiotics in our settings. Changes of first - line drugs should be based on the identification of isolated organisms and appropriate implementation of likely susceptible antibiotics would have a substantial impact on the outcomes.

\section{Conflict of Interest}

The authors declare that they have no conflict of interest.

\section{Acknowledgements}

We are grateful to Mrs Dang Thuy Hong for her contribution to performing laboratory tests.

\section{REFERENCES}

1. Yang $\mathrm{YN}$, Tseng HI, Yang $\mathrm{SN}$, et al. A strategy for reduction of antibiotic use in new patients admitted to a neonatal intensive care unit. Pediatr Neonatol 2012;53:245 - 51. 
2. Vergnano S, Sharland M, Kazembe $P$, et al.. Neonatal sepsis: an international perspective. Arch Dis Child Fetal Neonatal Ed. 2005

3. Kruse AY, Thieu Chuong do H, Phuong $\mathrm{CN}$, et al.. Neonatal bloodstream infections in a pediatric hospital in Vietnam: a cohort study. $J$ Trop Pediatr. 2013 Dec;59(6):483 - 8.

4. Peters L, Olson L, Khu DTK, et al. Multiple antibiotic resistance as a risk factor for mortality and prolonged hospital stay: A cohort study among neonatal intensive care patients with hospital - acquired infections caused by gram negative bacteria in Vietnam. PLoS One. 2019 May 8;14(5):e0215666.

5. Nguyen KV, Thi Do NT, Chandna A, et al. Antibiotic use and resistance in emerging economies: a situation analysis for Viet Nam. BMC Public Health. 2013 Dec 10;13:1158.

6. Patel JB. Approved Guideline, M - 02 e M 07. Clinical and Laboratory Standards Institute. Performance Standards for Antimicrobial Susceptibility Testing MS100-26. 2016.

7. Li JY, Chen SQ, Yan YY, et al. Identification and antimicrobial resistance of pathogens in neonatal septicemia in China - A meta - analysis. Int J Infect Dis. 2018 Jun;71:89 - 93.

8. Mularoni A, Madrid M, Azpeitia A, et al. The role of coagulase - negative staphylococci in early onset sepsis in a large European cohort of very low birth weight infants.

9. Fahmey SS. Early - onset sepsis in a neonatal intensive care unit in Beni Suef, Egypt: bacterial isolates and antibiotic resistance pattern. Korean J Pediatr. 2013 Aug;56(8):332 - 7.
10. Cailes, B.; Kortsalioudaki, C.; Buttery, J.; et al. Epidemiology of UK neonatal infections: The neonIN infection surveillance network. Arch. Dis. Child. - Fetal Neonatal Ed. 2018, 103, F547-F553.

11. Jean - Baptiste N, Benjamin DK Jr, Cohen - Wolkowiez M, et al. Coagulase negative staphylococcal infections in the neonatal intensive care unit. Infect Control Hosp Epidemiol. 2011 Jul;32(7):679 - 86.

12. Chaurasia S, Sivanandan S, Agarwal $\mathrm{R}$, et al. Neonatal sepsis in South Asia: huge burden and spiralling antimicrobial resistance. BMJ.2019 Jan 22;364:k5314.

13. Dat VQ, Vu HN, Nguyen The $H$, et al. Bacterial bloodstream infections in a tertiary infectious diseases hospital in Northern Vietnam: aetiology, drug resistance, and treatment outcome. BMC Infect Dis. $2017 \mathrm{Jul}$ 12;17(1):493.

14. Brooke JS. Stenotrophomonas maltophilia: an emerging global opportunistic pathogen. Clin Microbiol Rev. 2012 Jan;25(1):2 $-41$.

15. Li X, Ding X, Shi P, Zhu Y, et al. Clinical features and antimicrobial susceptibility profiles of culture - proven neonatal sepsis in a tertiary children's hospital, 2013 to 2017. Medicine (Baltimore). 2019 Mar;98(12):e14686. .

16. Nguyen $\mathrm{QH}$, Nguyen TK, Ho D, et al. Unnecessary antibiotic use for mild acute respiratory infections during 28 - day follow - up of 823 children under five in rural Vietnam. Trans R Soc Trop Med Hyg. 2011 Nov;105(11):628 - 36. 